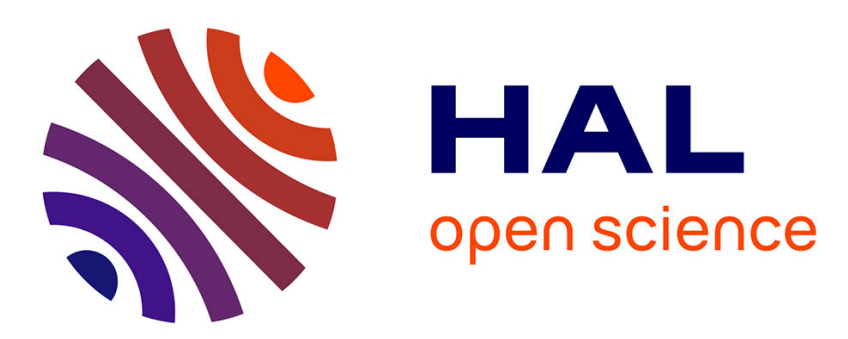

\title{
The Macroeconomic Performance of the Inflation Targeting Policy: An Approach Based on the Evolutionary Co-spectral Analysis
}

\author{
Zied Ftiti
}

\section{- To cite this version:}

Zied Ftiti. The Macroeconomic Performance of the Inflation Targeting Policy: An Approach Based on the Evolutionary Co-spectral Analysis. 2009. halshs-00422662

\section{HAL Id: halshs-00422662 \\ https://shs.hal.science/halshs-00422662}

Submitted on 8 Oct 2009

HAL is a multi-disciplinary open access archive for the deposit and dissemination of scientific research documents, whether they are published or not. The documents may come from teaching and research institutions in France or abroad, or from public or private research centers.
L'archive ouverte pluridisciplinaire HAL, est destinée au dépôt et à la diffusion de documents scientifiques de niveau recherche, publiés ou non, émanant des établissements d'enseignement et de recherche français ou étrangers, des laboratoires publics ou privés. 


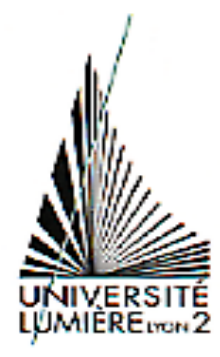

\section{DOCUMENTS DE TRAVAIL - WORKING PAPERS}

$$
\text { W.P. 09-18 }
$$

The Macroeconomic Performance of the Inflation Targeting Policy:

An Approach Based on the Evolutionary Co-spectral Analysis

\section{Zied Ftiti}

Août 2009

GATE Groupe d'Analyse et de Théorie Économique UMR 5824 du CNRS

93 chemin des Mouilles - 69130 Écully - France

B.P. $167-69131$ Écully Cedex

Tél. +33 (0)4 72866060 - Fax +33 (0)4 72866090

Messagerie électronique gate@gate.cnrs.fr

Serveur Web : www.gate.cnrs.fr 


\title{
The Macroeconomic Performance of the Inflation Targeting Policy: An Approach Based on the Evolutionary Co-spectral Analysis
}

\author{
FTITI Zied*
}

3 August

\begin{abstract}
This paper proposes a new methodology to check the economic performance of a monetary policy and in particular the inflation targeting policy (ITP). The main idea of this work is to consider the ITP as economically efficient when it generates a stable monetary environment. The latter is considered as stable when a long-run equilibrium exists to which the paths of economic variables (inflation rate, interest rate and GDP growth) converge. The convergence of the variables' paths implies that these variables are more predictable and implies a lower degree of uncertainty in the economic environment. To measure the degree of convergence between economic variables, we propose, in this paper, a dynamic time-varying variable presented in the frequency approach named cohesion. This variable is estimated from the evolutionary co-spectral theory as defined by Priestley and Tong (1973) and Priestley (1988-1996). We apply this theory to the measure of cohesion presented by Croux et al (2001) to obtain a dynamic time-varying measure. In the last step of the study, we apply the Bai and Perron test (1998-2003b) to determine the change in the cohesion path. The results show that the implementation of the ITP generates a high degree of convergence between economic series that implies less uncertainty into the monetary environment. We conclude that the inflation targeting generates a stable monetary environment. This result allows us to conclude that the ITP is relevant in the case of industrialized countries.

JEL: C16, E52, E63.

Mots-clés : Inflation Targeting, Co-Spectral Analysis, Cohesion, Stability Environment, Economic Performance and Structural Change.
\end{abstract}

\section{Introduction}

Since the early 1990s, a monetary policy strategy known as "inflation targeting (IT)" has been formally adopted by a number of industrialized countries. Under this approach, a monetary authority officially announces an inflation target level or target range, which is generally consistent with low or zero inflation, to be achieved within a specified time frame. This monetary policy was adopted without inherent theory. This lateness has attracted some debates. Among them, we find the recent debate about the macroeconomic performance of the inflation targeting policy (ITP). There has been a growing interest, both theoretical and empirical, in this subject. These researches are separated into two majors fronts. The first, based on the effect of this

*ftiti@gate.cnrs.fr; PhD Student at the University of Lyon, (GATE), CNRS, UMR 5824, Ecully, F-6913, France and University of Tunis: (FIESTA) - High Business School (ISG-Tunis, Tunisia). 
policy on the output, finds inspiration in the researches of Buckel et al (2003) and Mollick et al (2008)... They consider the ITP as a relevant policy that leads to the best economic performance when it generates a positive impact on the output. The second front is concerned with the impact of the ITP on some economic variables. ${ }^{1}$ This front of research finds inspiration in the works of Mishkin and Posen (1997), Bernanke et al (1999), Honda (2000) and Ball and Sheridan (2003)... The idea behind these pieces of research consists of checking the effect of IT implementation on the evolution of these variables. In the case when their paths become stable after the ITP intervention, we can characterize this policy as relevant.

These works reach different conclusions. Some of them show the efficiency of the ITP and justify its relevance. The others show that the ITP had no impact on the dynamic of the economic variables. We suggest that the different conclusions are the result of a less rigorous econometric approach. In the first kind of work, the authors test the effect of the ITP only on the output. So, in the case where this policy generates a bad consequence for the evolution of other variables, this econometric approach can not detect it. Indeed, in the second kind of work, most of the researchers model the studied variables before the inflation targeting adoption by an $\mathrm{ARCH}$, ARMA, GARCH or VAR model... Then, they generate a realization of these variables during the IT intervention. After that, they test the significant difference between the observed series and the predicted one during the period of intervention. In these types of works, we notice two problems. The first one is related to a misspecification of the variables' processes. The second one concerns the construction of the test. Indeed, authors test whether the date of the inflation targeting adoption is a date of structural break or not. In other words, they didn't test whether the ITP had an effect on the stabilization of the economic variables. We suggest, in the case of the effect of ITP on the economic series, that the change in their paths can occur before or after the intervention of the policy.

In this paper, we propose a new methodology that allows us to judge the economic performance of the ITP. It is an original methodology allowing us to brighten this debate. In this work, we consider the monetary policy to be relevant and economically efficient when it ensures a stable monetary environment. Indeed, a stable monetary environment implies a low level of uncertainty. This low level of uncertainty traduces the predictability of the economic variables and a high level of convergence between the expectations of economic agents and those of the policy-makers. We consider the monetary environment as stable when the economic variables (interest rate, inflation rate, output growth $)^{2}$ converge in the long run to an equilibrium state. In other words, the interaction between the variables must be higher under the relevant monetary policy, which traduces a low level of uncertainty and a predictable environment. These variables must have the same response to economic shocks.

The methodology that we present above to check the economic performance of the monetary policy and particularly the relevance of the inflation targeting is based on the literature about long-run growth ${ }^{3}$ and macroeconomic stability. Business cycle and growth theories have traditionally been treated as unrelated areas of macroeconomics. Three papers published in the early 1980s, however, changed this perspective. First, Nelson and Plosser (1982) presented evidence

\footnotetext{
${ }^{1}$ Like the interest rate, the inflation rate, the output and the exchange rate.

${ }^{2}$ We choose these three variables because, in most of the cases of industrial inflation targeting countries, these three variables are present in the monetary rule of this policy.

${ }^{3}$ In the literature, the long-run growth is considered as a proxy for economic performance. In other words, a country that provides a high long-run growth is qualified by performing economically.
} 
that movement in the GNP tends to be permanent, and, second, Kydland and Prescott (1982) offered new models for analysing economic fluctuations that integrated growth and business cycle theory. According to these models, output fluctuations are induced by stochastic variations in technology. In more recent contributions to this literature, Robert King et al (1988) incorporate endogenous growth into a real business cycle model, with the result that the temporary disturbances to production possibilities can have permanent effects on the path of the output. During this period, little attention was paid to the effect of business cycle volatilities on growth. This literature, in the middle of the 1980s, developed a positive relationship ${ }^{4}$ between long-run growth and business cycle volatilities. Among this research, we find Kormendi and Meguire (1985), Black and Fisher (1987), Grier and Tullock (1989), Caballero and Hammour (1994), Canton (1996) and Caporale and McKiernan (1996). However, in the middle of the 1990s, this literature developed a contrary conclusion. In other words, the relationship that is proved by recent works shows that the volatilities of the business cycle had a negative impact on the longrun growth. Among this research, we find Aizenman and Marion (1993), Ramey and Ramey (1995), Ho (1996), Elbadawi and Hebbel (1998), Sanchez-Robles (1998), Lensink et al (1999), Martin and Rogers (2000), Beaudry et al (2001), Kneller and Young (2001), Ismihan et al (2003), Winkler (2003), Blackburn and Pellonni (2005) and Stiroh (2006). This second front of research criticizes the first one regarding the fact that they are based on the traditional growth theory. Indeed, they explain that the conclusion of the first front of research was the result of some strong hypotheses. Indeed, they study this relationship by considering the investment as the most important transmission canal. ${ }^{5}$ The famous paper of Ramey and Ramey (1995) broke with the idea that considers the investment to have a main role in the relationship between long-run growth and cycle volatilities. Other research, such as that of Fisher (1993), Turovsky (1993) and Beaudry et al (2001), studies the effect of an instable monetary environment on the economic performance. They show that a stable monetary environment is favourable to a good economic performance.

With the purpose of measuring the interaction between economic variables and their degrees of convergence, we use some notions such as co-movement ${ }^{6}$, precisely the coherence function and/or the cohesion ${ }^{7}$ function. Our empirical objective is to measure the movement of three time series. So, we use the theory of evolutionary co-spectral analysis (Priestley and Tong 1973) and Priestley (1988-1996). We construct a time-varying variable named cohesion that allows us to determine the long-run relationship between times series. ${ }^{8}$ The results show that the inflation rate, the interest rate and the growth rate converge more during the period of the inflation targeting policy. This implies that the monetary environment is stable during this policy. So, we conclude the relevance and the economic performance of the inflation targeting policy.

Our paper presents two major contributions. The first one concerns the theoretical methodology we followed to check the performance of a monetary policy based on the stability of the monetary environment. To our knowledge, there are no work that follow this methodology. The

\footnotetext{
${ }^{4}$ According to these researchers, they find that, when the cycles are volatile, the long-run growth is high.

${ }^{5}$ Their argument of the positive relationship between business cycle volatilities is as follows: under an uncertain environment, the agents behave prudently and they will use precautionary savings. However, if the savings increase, the investment will increase.

${ }^{6}$ The co-movement notion refers to the case of a bivariate process.

${ }^{7}$ The cohesion function refers to the case of a multivariate process.

${ }^{8}$ The Cohesion function replace the coherence function when the number of time series are superior to 2
} 
second one concerns the original empirical methodology.

The paper is organized as follows. Section 2 presents the empirical analysis. Section 3 explains the data and shows the results. Then, section 4 presents interpretations. The last section encompasses the conclusion.

\section{Empirical Analysis}

As mentioned in the introduction, our objective consists of measuring the degree of stability in the monetary environment. In most of the cases, this environment is represented by three economic indicators: inflation rate, interest rate and GDP growth. ${ }^{9}$ In our analysis, we consider this environment to be stable if the paths of these variables have the same movement in the long run. In other words, if a long-run equilibrium state exists in this environment, to which the paths of these variables converge, we then have stability. This movement between these variables is measured by the cohesion function. We propose a time-varying measure of this variable. So, we adopt the theory of the evolutionary co-spectral analysis as defined by Priestley and Tong (1973), which allows us to determine the interaction between non-stationary processes in two dimensions: the time dimension and the frequency one. After the estimation of all the pairs' relationships between the three variables (coherence), we construct the cohesion variable as a weighted average of all the coherence pairs. The last step is to check the evolution of the cohesion path. In others words, our objective is to verify whether the cohesion path has increased during the period of inflation targeting adoption or not. For this reason, we adopt the Bai and Perron test (1998-2003b), which allows us to identify a multiple endogenous break point. In this section, we start by presenting the evolutionary co-spectral theory and the estimation method of the density function. Then, we will present the estimation of the coherence function. After that, we present the measure of the cohesion function. In the last step, we present the test of Bai and Perron (1998-2003b).

\subsection{Theory of the evolutionary Co-spectral (Priestley and Tong : 1973)}

According to Priestley (1965), a non-stationary discrete ${ }^{10}$ process or a continuous ${ }^{11}$ process can be written as equation (1). Priestley and Tong (1973) extend the theory of the evolutionary spectral analysis of Priestley (1965-1966), presented by Ftiti and Essaadi (2008), to the case of a bivariate non-stationary process. In this sub-section, we present this theory.

Consider, for example, a bivariate continuous parameter process $\{X(t), Y(t)\}$, in which each component is an oscillatory process. Each component can be written as follows:

$$
X_{t}=\int_{-\infty}^{+\infty} A_{t, X}\left(w_{1}\right) e^{i w t} d Z_{X}\left(w_{1}\right) .
$$

\footnotetext{
${ }^{9}$ We choose only these three variables because, in the case of an inflation targeting policy, the reaction function that represents the ITP includes, in the case of industrial countries, only these three variables.

${ }^{10} \mathrm{~A}$ discrete process corresponds to a process of which the value of $\mathrm{T}$ is countable. Indeed, a time series is considered as a discrete process.

${ }^{11} \mathrm{~A}$ continuous process is a process used to describe the physical signal.
} 


$$
Y_{t}=\int_{-\infty}^{+\infty} A_{t, Y}\left(w_{2}\right) e^{i w t} d Z_{Y}\left(w_{2}\right)
$$

where

$$
\begin{aligned}
E\left[d Z_{X}\left(w_{1}\right) d Z_{X}^{*}\left(w_{2}\right)\right] & =E\left[d Z_{Y}\left(w_{1}\right) d Z_{Y}^{*}\left(w_{2}\right)\right] \\
& =\left[d Z_{X}\left(w_{1}\right) d Z_{Y}^{*}\left(w_{2}\right)\right]=0
\end{aligned}
$$

for $w_{1}=w_{2}$

$$
E\left[\left|d Z_{X}\left(w_{1}\right)\right|^{2}\right]=d \mu x x\left(w_{1}\right) \quad E\left[\left|d Z_{Y}\left(w_{1}\right)\right|^{2}\right]=d \mu Y Y\left(w_{1}\right)
$$

and, $E\left[d Z_{X}\left(w_{1}\right) d Z_{Y}^{*}\left(w_{1}\right)\right]=d \mu X Y\left(w_{1}\right)$

with, [.]* denoting the conjugate function of [.].

Let, $F_{X}, F_{Y}$ denote respectively the families of oscillatory functions as: $\left\{\phi_{t, X}\left(w_{1}\right) \equiv A_{t, X}\left(w_{1}\right) e^{i w t}\right\}$, $\left\{\phi_{t, Y}\left(w_{1}\right) \equiv A_{t, Y}\left(w_{1}\right) e^{i w t}\right\}$. Priestley and Tong (1973) define the evolutionary power crossspectrum at time $\mathrm{t}$ with respect to the families $F_{X}, F_{Y}, d H_{t, X Y}$ by

$$
d H_{t, X Y}=A_{t, X} A_{t, Y}^{*} d \mu_{X Y}(w)
$$

Further, if $\{X(t), Y(t)\}$ is a bivariate stationary process, so that $F_{X}$ and $F_{Y}$ may be chosen to be the family of complex exponentials, namely $F_{x} \equiv F_{Y} \equiv\left\{e^{i w t}\right\}, d H_{t, X Y}(w)$ reduces to the classical definition of the cross-spectrum. Thus, for each t, we may write

$$
d H_{t, X Y}=E\left[A_{t, X} d Z_{X}(w) A_{t, Y}^{*} d Z_{Y}^{*}(w)\right]
$$

Priestley and Tong (1973) extend the above relation to the case of a non-stationary bivariate process where the amplitudes are time-dependent; correspondingly, the cross-spectrum is also time-dependent. Clearly, $d H_{t, X Y}$ is complex-valued, and, by virtue of the Cauchy-Schwarz equality, we have immediately that

$$
\left|d H_{t, X Y}\right|^{2} \leq d H_{t, X X} d H_{t, Y Y}: \forall t, w
$$

If the measure $\mu_{X Y}(w)$ is absolutely continuous with respect to the Lebesgue measure, we may write, for each $\mathrm{t}$ :

$$
d H_{t, X Y}=h_{t, X Y}(w) d w
$$

and $h_{t, X Y}(w) d w$ may then be termed the evolutionary cross-spectral density function.

\subsection{Estimation of the evolutionary Co-spectral density function}

The evolutionary cross-spectral density function estimation, which we develop here, is an extension of Priestley and Tong (1973) from the estimation of the evolutionary spectral density function in the univariate case, such as developed by Priestley (1965-1966). In our analysis, we develop the two cases: the discrete case and the continuous process. 


\subsubsection{Case of continuos process}

Suppose we are given a sample record of a continuous parameter process $\{X(t), Y(t)\}$ say for $t \in\left[0, T_{0}\right]$. One method of estimating the evolutionary cross-spectral density function $h_{t, X Y}(w)$ is based on an extension of the method for dealing with the univariate case. This latter is based is the notion of a filter (window). ${ }^{12}$ So, the estimation of $h_{t, X Y}(w)$ is relevant to two steps:

- First, we pass the data through a linear filter centred on frequency $w_{0}$, say yielding an output $U_{X}(t, w)$ and $U_{Y}(t, w)$

- Second, we compute a weighted average of $\left|U_{X}(t, w) U_{Y}(t, w)\right|$ in the neighbourhood of the time point $\mathrm{t}$ to provide an estimation of the local power density at frequency $w_{0}$.

In the object of the generalization, we note, in the following expression, that $w=w_{0}$. Let $\{g(u)\}$ be a filter whose transfer function and, respecting the usual conditions defined by Priestley (1965), w consists of:

$$
2 \pi \sum_{u \in Z}|g(u)|^{2}=\int_{-\infty}^{+\infty}|\Gamma(w)|^{2} d w=1
$$

with

$\Gamma(w)=\int_{-\infty}^{+\infty} g(u) e^{-i w u} d u$

filter $\{g(u)\}$ is double integrable. The width of the filter is described as:

$$
B_{g}=\int_{-\infty}^{+\infty}|u||g(u)| d u
$$

The filter $\{g(u)\}$ is chosen such that its width $B_{g}$ is much smaller than $B_{X}: B_{g} \ll \min \left(B_{X}, B_{Y}\right) \ll$ $T_{0}$ (with $\left(B_{X}, B_{Y}\right)$ meaning the maximum interval of the local stationarity) and $B_{g} \ll T$ (with T:sample length).

For any frequency $w$, we can write that:

$$
\begin{aligned}
U_{X}(t, w) & =\int_{t-T_{0}}^{t} g(u) X(t-u) e^{-i w(t-u)} d u \simeq \int_{-\infty}^{+\infty} g(u) X(t-u) e^{-i w(t-u)} d u \\
U_{Y}(t, w) & =\int_{t-T_{0}}^{t} g(u) Y(t-u) e^{-i w(t-u)} d u \simeq \int_{-\infty}^{+\infty} g(u) Y(t-u) e^{-i w(t-u)} d u
\end{aligned}
$$

Next, let $W_{T^{\prime}}(t)$ be a weight function, depending on the parameter $T^{\prime}$ (which characterizes its "width") and satisfying the usual conditions (Priestley 1965) that we note below:

a $W_{T^{\prime}}(t) \geq 0$, for all $T^{\prime}$

b $W_{T^{\prime}}(t)$ converge to zero if $|t| \rightsquigarrow \infty$, for all $T^{\prime}$

c $\int_{-\infty}^{+\infty} W_{T^{\prime}}(t) d t=1$, for all $T^{\prime}$

d $\int_{-\infty}^{+\infty}\left\{W_{T^{\prime}}(t)\right\}^{2} d t \prec \infty$, for all $T^{\prime}$.

e A constant C exists, such as: $\operatorname{Lim}\left\{T^{\prime} \int_{-\infty}^{+\infty}\left|W_{T^{\prime}}(\lambda)\right|^{2} d \lambda\right\}=C$ with $W_{T^{\prime}}(\lambda)=\int_{-\infty}^{+\infty} e^{-i \lambda t} W_{T^{\prime}}(t)$

\footnotetext{
${ }^{12}$ For more details see Ftiti and Essaadi (2008).
} 
According to these two filters, we can estimate the evolutionary cross-spectral density function $h_{t, X Y}(w)$ for each frequency $w$ :

$$
\hat{h}_{t, X Y}(w)=\int_{t-T_{0}}^{t} W_{T^{\prime}}(u)\left[U_{X}((t-u), w) U_{Y}^{*}((t-u), w)\right] d u
$$

The limits of the integral in (11) can be replaced by $(-\infty,+\infty) . \hat{h}_{t, X Y}(w)$ is an unbiased estimate of the average value of $h_{t, X Y}(w)$ in the neighbourhood of $t$. An investigation into the sampling proprieties of $\hat{h}_{t, X Y}(w)$ has been carried out. Although in many ways the method of investigation is similar to that of Priestley (1966), both the calculations and results are much lengthier. In this paper, we summarize the main results. ${ }^{13}$

$$
\begin{gathered}
E\left[\hat{h}_{t, X Y}(w)\right] \approx \bar{h}_{t, X Y}(w) \\
\bar{h}_{t, X Y}(w+\theta)=\int_{-\infty}^{+\infty} W_{T^{\prime}}(u) h_{t-u, X Y}(w+\theta) d u
\end{gathered}
$$

The variance

$$
\operatorname{var}\left(h_{t, X Y}\right) \approx\left(C / T^{\prime}\right)\left\{\int_{-\infty}^{+\infty}|\Gamma(\theta)|^{4} d \theta\right\} \widetilde{f}_{t}^{2}(w)
$$

with,

$$
\widetilde{f}_{t}^{2}(w)=\frac{\int_{-\infty}^{+\infty} f_{t-u}^{2}\left\{W_{\Gamma}(u)\right\}^{2} d u}{\int_{-\infty}^{+\infty}\left\{W_{\Gamma}(u)\right\}^{2} d u}
$$

Priestley and Tong (1973) showed that the covariance between $\hat{h}_{t, X Y}(w)$ and $\hat{h}_{t, X Y}\left(w^{\prime}\right)$ is effectively zero if either:

(i) $\left|w \pm w^{\prime}\right|$ are enough wide such as $\left|w_{1} \pm w_{2}\right| \gg$ to the band width $|\Gamma(w)|^{2}$.

(ii) $|S-t|$ more broader that the function of $\{w(u)\}$

If these conditions are satisfied according to Priestley (1965), Priestley and Tong (1973) adopt the following assumption that:

$$
E\left\{\hat{h}_{t, X Y}(w)\right\} \approx h_{t, X Y}(w)
$$

\subsubsection{Case of discrete process}

According to Priestley (1965-66), the theory presented above is applied to the discrete process by simply replacing the limits of integral $(-\infty,+\infty)$ with $(-\pi,+\pi)$ and the integral of equations 9,10 and 11 with the sum. The corresponding function of these equations is presented below respectively in the equations 18,19 and 20 .

In our analysis, we are interested in the analysis of three time series (inflation, interest rate and GDP). So, we recapitulate in this part the procedure to estimate the evolutionary cross-spectral density function. Let a non-stationary discrete bivariate process $\{X(t), Y(t)\}$ have the Gramer representation for each $-\pi \prec w \prec+\pi$

\footnotetext{
${ }^{13}$ For more details on the estimators proprieties', see Tong (1972).
} 


$$
X_{t}=\int_{-\pi}^{+\pi} A_{t, X} e^{i w t} d Z_{X}(w) \text { et } Y_{t}=\int_{-\pi}^{+\pi} A_{t, Y} e^{i w t} d Z_{Y}(w)
$$

with

$$
\begin{aligned}
E\left[d Z_{X}\left(w_{1}\right) d Z_{X}^{*}\left(w_{2}\right)\right] & =E\left[d Z_{Y}\left(w_{1}\right) d Z_{Y}^{*}\left(w_{2}\right)\right] \\
& =\left[d Z_{X}\left(w_{1}\right) d Z_{Y}^{*}\left(w_{2}\right)\right]=0
\end{aligned}
$$

for $w_{1}=w_{2}$

$$
E\left[\left|d Z_{X}\left(w_{1}\right)\right|^{2}\right]=d \mu x x\left(w_{1}\right) \quad E\left[\left|d Z_{Y}\left(w_{1}\right)\right|^{2}\right]=d \mu Y Y\left(w_{1}\right)
$$

and, $E\left[d Z_{X}\left(w_{1}\right) d Z_{Y}^{*}\left(w_{1}\right)\right]=d \mu X Y\left(w_{1}\right)$

By virtue of the Cauchy-Schwarz inequality, we can write that:

$\left|d H_{t, X Y}\right|^{2} \leq d H_{t, X X} d H_{t, Y Y}:$ for all $\mathrm{t}$ and $\mathrm{w}$

and, $d H_{t, X Y}=h_{t, X Y}(w) d w$

where $h_{t, X Y}$ may then be termed the evolutionary cross-spectral density function.

The estimation of the evolutionary cross-spectral density function needs two filters. For the discrete univariate process, Priestley (1966) gives two relevant windows. These are relevant filters and they are tested by several researchers such as Ahamada and Boutahar (2002), Ben Aissa (2004) and Ftiti and Essaadi (2008). For the discrete bivariate process, Priestley and Tong (1973) adopt the same choice that:

$$
g_{u}=\left\{\begin{array}{cc}
1 /(2 \sqrt{h \pi}) & \text { if }|u| \leq h \\
0 & \text { if }|u|>h
\end{array} \mid\right.
$$

et

$$
w_{v}=\left\{\begin{array}{cc}
1 / T^{\prime} & \text { if }|v| \leq T^{\prime} / 2 \\
0 & \text { if }|v|>T^{\prime} / 2
\end{array} \mid\right.
$$

Then, the estimation of the evolutionary cross-spectral density function is as follows:

$$
\hat{h}_{t, X Y}=\sum_{\nu \in Z} W_{T^{\prime}}(\nu) U_{X}(w, t-\nu) U_{Y}(w, t-\nu)
$$

with,

$$
\begin{aligned}
& U_{X}(t, w)=\sum_{u \in Z} g(u) X(t-u) e^{i w(t-u)} d u \\
& U_{Y}(t, w)=\sum_{u \in Z} g(u) Y(t-u) e^{i w(t-u)} d u
\end{aligned}
$$

In this paper, we take $h=7$ and $T^{\prime}=20$. We make the same choice ${ }^{14}$ as do Artis et al. (1992), Priestley (1995), Ahamada and Boutahar (2002), Ben Aissa and Ahamada (2004), Essaadi and Boutahar (2008) and Ftiti and Essaadi (2008).

\footnotetext{
${ }^{14}$ This choice of values is justified by the fact that they respect the conditions (i) and (ii).
} 
According to Priestley (1988), if we have $E(\hat{h}(w)) \approx h_{t}(w), \operatorname{var}(\hat{h}(w))$ decreases when $T^{\prime}$ increases $\forall\left(t_{1}, t_{2}\right), \forall\left(w_{1}, w_{2}\right), \operatorname{cov}\left(\hat{h}_{t 1}\left(w_{1}\right), \hat{h}_{t 1}\left(w_{2}\right)\right)=0$, if at least one of the conditions (i) or (ii) is satisfied.

\subsection{Coherence and cohesion}

\subsubsection{Coherence function}

According to Priestley and Tong (1973), the evolutionary cross-spectral density function may be written as:

$$
\begin{gathered}
h_{t, X Y}=C_{t, X Y}-i Q_{t, X Y}(w) \\
C_{t, X Y}(w)=R\left\{h_{X Y}\left(w_{j}, t\right)\right\} \\
Q_{t, X Y}(w)=\operatorname{Im}\left\{h_{X Y}(w, t)\right\}
\end{gathered}
$$

and the real-valued functions $C_{t, X Y}(w)$ and $Q_{t, X Y}(w)$ termed the evolutionary co-spectrum and the evolutionary quadrature spectrum, respectively. If the measures $\mu_{X X}(w)$ and $\mu_{Y Y}(w)$ are absolutely continuous, Priestley and Tong (1973) similarly define the evolutionary auto-spectral density functions, $h_{X X}\left(w_{j}, t\right), h_{Y Y}\left(w_{j}, t\right) .{ }^{15}$ The coherency function is defined by the following expression:

$$
\begin{aligned}
C_{t, X Y}(w) & =\frac{\left|h_{t, X Y}(w)\right|}{\left\{h_{t, X X}(w) h_{t, Y Y}(w)\right\}^{1 / 2}} \\
& =\frac{\left|E\left[d Z_{Y}(w) d Z^{*}(w)\right]\right|}{\left\{E\left|d Z_{X}(w)\right|^{2} E\left|d Z_{Y}(w)\right|^{2}\right\}^{1 / 2}}
\end{aligned}
$$

Priestley and Tong (1973) interpret $C_{t, X Y}(w)$ as the modulus of the correlation coefficient between $d Z_{X}(w), d Z_{Y}(w)$ or, more generally, as a measure of the linear relationship between corresponding components at frequency $\mathrm{w}$ in the processes $\{Y(t)\}$ and $\{X(t)\}$.

The estimation of the coherency function is based on the estimation of the cross-spectral density function between two processes $\{Y(t)\}$ and $\{X(t)\}$ and the estimation of the auto-spectral density function of each process. So, the estimation coherency can be written as follows:

$$
\hat{C}_{t, X Y}(w)=\frac{\left|\hat{h}_{t, X Y}(w)\right|}{\left\{\hat{h}_{t, X X}(w) \hat{h}_{t, Y Y}(w)\right\}^{1 / 2}}
$$

\subsubsection{Cohesion function}

In the above section, we presented a coherency function that allows us to measure the interaction between two processes; however, in the case when we try to measure the interaction and the relationship between more than two processes, this measure cannot be used. Croux et al (2001) define a measure, which they name cohesion, that can determine this when $n \succ 2$, with $\mathrm{n}$ the number of processes. According to Croux et al (2001), this measure is based on the bivariate case. In other words, it is based on the coherency function. The cohesion function, as presented

\footnotetext{
${ }^{15}$ For more details see Ftiti and Essaadi (2008).
} 
by Croux and al (2001), equals the weighted average of the dynamic correlation between all possible pairs of series. It can be written as follows:

$$
\operatorname{coh}_{X}(\lambda)=\frac{\sum_{i \neq j} w_{i} w_{j} \rho_{X i X j}(\lambda)}{\sum_{i \neq j} w_{i} w_{j}}
$$

with $\rho_{X i X j}(\lambda)$ the dynamic correlation between two pairs of series and the weight of series $i$. We adopt the same function of coherence as defined by Croux et al (2001). However, our variable is time-varying. Indeed, we replace the dynamic correlation defined by Croux et al (2001), which is independent of time in the above function, with our measure of coherency presented in the above section. So, the measure that we present in this paper can be written as follows:

$$
\operatorname{coh}_{t, X Y}(w)=\frac{\sum_{i \neq j} W_{i} W_{j} C_{t, X Y}(w)}{\sum_{i \neq j} W_{i} W_{j}}
$$

Another difference according to Croux et al (2001) consists of the weight accorded to the series. In our analysis, we accord the same weight to each series, which is equal to one. We interpret this measure by the degree of convergence in frequency w of the economic series.

\subsection{Detection of multiple structural break points}

At this step of the analysis, we can estimate the cohesion function in all the sample sizes. However, we must employ an empirical method that detects the change in the relationship between economic series. For this purpose, we adopt the Bai and Perron (1998-2003b) test. This has some advantages. It allows us the opportunity to detect multiple break points in a time series and their occurrence endogenously. We use GAUSS software and we obtain the estimate by running the code created by Bai and Perron $(1998,2003 b) .{ }^{16}$

\subsubsection{The model and Estimators}

Our objective is to detect the break point in a cohesion function, so we consider the following mean shift model:

$$
\begin{aligned}
\operatorname{coh}\left(w_{j}, t\right) & =\mu_{1}+\mu_{t} \quad \text { for } \quad t=1, \ldots, T_{1} \\
\operatorname{coh}\left(w_{j}, t\right) & =\mu_{2}+\mu_{t} \quad \text { for } \quad t=T_{1}, \ldots, T_{2} \\
\vdots &
\end{aligned}
$$

with $j=1, \ldots, m+1, T_{0}=0, T_{m+1}=T$. T is the sample size, $\mu_{j}$ are the means and $\mu_{t}$ is the disturbance at time t. The break points $\left(T_{1}, \ldots, T_{m}\right)$ are treated as unknown. According to Bai and Perron (1998-2003b), the estimation of the coefficients of regressors is based on minimizing the sum of squared residuals according to the following form:

$\operatorname{Min} \sum_{j=1}^{m+1} \sum_{t=T_{i-1}+1}\left(\operatorname{coh}_{t}-\mu_{j}\right)^{2}$

Let $\hat{c o h}\left(\left\{T_{j}\right\}\right)$ denote the resulting estimate. The sum of squared residuals for all partitions

\footnotetext{
${ }^{16}$ The code is available on the Perron home page: http://people.bu.edu/perron/.
} 
$\left(T_{1}, \ldots, T_{m}\right)$, which correspond to these estimators, is:

$S_{T}\left(T_{1}, \ldots, T_{m}\right)=\left(X-\hat{X}\left(\left\{T_{j}\right\}\right)^{\prime}\left(X-\hat{X}\left(\left\{T_{j}\right\}\right)\right.\right.$

The estimated break points are presented as:

$$
\left(\hat{T}_{1}, \ldots, \hat{T}_{m}\right)=\arg _{\left(T_{1}, \ldots, T_{m}\right)} \min S_{T}\left(T_{1}, \ldots, T_{m}\right)
$$

\subsubsection{The statistic test and the model selection criteria}

In order to determine the number of break points in the cohesion series, we adopt the selection criteria. The idea behind these criteria is as follows: a supplementary break point decreases the sum of squared residuals in the regression. So, it is useful to punish the addition break point. We find three types of criteria information: the Aikake (AIC), the Bayesian (BIC) and the Schwartz (LWZ). Perron (1997) realized a simulation experimentation to evaluate which is the relevant criterion. The results are favourable for the LWZ and BIC criteria but show a major performance of the LWZ. So, in our analysis, we adopt the Schwartz criterion (LWZ), which is modified by Liu, Wu and Zidek (1997), to detect the number of break points. This criterion is defined as follows:

$L W Z(m)=\operatorname{Ln}\left(S_{T}\left(\hat{T}_{1}, \ldots, \hat{T}_{m}\right) /\left(T-P^{*}\right)\right)+\left(p^{*} / T\right) c_{0}(\operatorname{Ln}(T))^{2+\delta_{0}}$ where $\left(p^{*} / T\right) c_{0}(\operatorname{Ln}(T))^{2+\delta_{0}}$ is a penalty factor.

The results of the simulation exercise of Bai and Perron (1998-2003b) show that it is relevant to use the following measure: $\delta_{0}=1$ et $c_{0}=0.299$.

Bai and Perron (1998-2003b) present some asymptotic critical values for the arbitrary small positive number $\epsilon$, and the maximum number of breaks (M). For example, $\epsilon=0.05(M=9), \epsilon=$ $0.10(M=8), \epsilon=0.15(M=5), \epsilon=0.2(M=3), \epsilon=0.25(M=2)$, In our work, we follow Bai and Perron (1998): $\epsilon=0.15(M=5)$.

\section{Data and Results}

\subsection{Data}

In our analysis, we try to measure the long-term relationship between three time series: inflation rate, interest rate and growth rate of GDP. The objective of our study is to evaluate the macroeconomic performance of inflation targeting. We try to pursue the cases studied by Ftiti and Essaadi (2008) to judge the efficiency of the inflation targeting policy for four industrials countries: New Zealand, Canada, the United Kingdom and Sweden. However, interest rate and GDP data are not available from 1962 in the case of Sweden and New Zealand, which is why we focus in this work on the cases of Canada and the United Kingdom.

In our study, we need the inflation rate, the interest rate and the growth of GDP. The inflation rate is calculated from the consumer price index according to the following equation:

$$
i n f_{t}=\frac{I P C_{t}-I P C_{t-4}}{I P C_{t-4}}
$$

The growth of GDP is calculated from the GDP series according to the following equation:

$$
Y=\frac{g d p_{t}-g d p_{t-4}}{g d p_{t-4}}
$$


The consumer price index, the interest rate and the GDP for each country are obtained from the International Financial Statistics published by IMF. These data are collected from the third quarter of 1961 to the fourth quarter of 2006. However, according to (28) and (29), we lose four observations, which is why we take the data for the three series from the third quarter of 1962 to the fourth quarter of 2006. Note that, for the evolutionary spectral estimation, we lose ten observations at the beginning and at the end of the sample. Thus, we have at our disposal 157 observations.

In order to respect the conditions (i) and (ii), we choose $\left\{t_{i}\right\}$ and $\left\{t_{j}\right\}$ as follows: $t_{i}=\{18+20 i\}_{i=1}^{I}$ where $I=\left[\frac{T}{20}\right]$ and $\mathrm{T}$ the sample size. $w j=\left\{\frac{\pi}{20}(1+3(j-1))\right\}_{j=1}^{T}$

To respect condition (ii), we inspect the instability in these frequencies: $\frac{\pi}{20}, \frac{4 \pi}{20}, \frac{7 \pi}{20}, \frac{10 \pi}{20}, \frac{13 \pi}{20}, \frac{16 \pi}{20}$ and $\frac{19 \pi}{20}$.

\section{$3.2 \quad$ Results}

Before presenting the results of our methodology, let us remind ourselves of it. Our methodological analysis consists of measuring the relationship between three economic series. This relationship is calculated from the cohesion function that is, as presented in the second section, calculated from the coherence function. The latter is calculated from the theory of the evolutionary spectral analysis of a discrete process as defined by Priestley and Tong (1973). The estimation of the coherency function was performed according to a code we have developed with MATLAB software. After that, we developed a sub-program that allows us to calculate the cohesion function according to a simple mathematical operation (see equation 27).

The results are summarised in four tables and four graphics presented respectively in Appendix 1 and 2 .

\section{Interpretations}

Graphics 2 and 4 give us information about the nature of the relationship between the interest rate, inflation rate and GDP growth, respectively for the cases of Canada and the United Kingdom, for all frequencies (7). However, graphics 1 and 3 represent the path of the relationship between these variables only for two frequencies, $\frac{\pi}{20}$ and $\frac{4 \pi}{20} .17$ The first one represents the longrun relationship (10 years) and the second one represents the medium relationship (5 years). As mentioned above, we will be interested only in the long-run relationship. In other words, we will focus in our analysis on the cohesion detected for the frequency $\frac{\pi}{20}$. According to our graphics, we detect a change in the path of the long-run relationship between the variables beginning with the $100^{t h}$ observation (nearly from 1990). To check our insight graphics, we refer to the Bai and Perron (1998-2003b) test, which informs us about the date of significant change occurrence in the cohesion variable. The results of the Bai and Perron (1998-2003b) test for the cases of Canada and the United Kingdom are summarized respectively in Tab. 1 and Tab. 3. In long-run cohesion, we identify two break points in each case (Tab. 1 and Tab. 3).

In the case of Canada, we detect a first break in the first quarter of 1975 (Tab. 1). Before this date, the cohesion between the three economic variables has a lower level, equal to 0.12. This low level of cohesion between the inflation rate, interest rate and GDP growth implies a frail

\footnotetext{
${ }^{17} T_{i}=\frac{2 \pi}{\lambda_{i}}$, with $i=1, \ldots, 7, \lambda$ representing the seven frequencies that we study.
} 
relationship. We can say that these variables have a divergent path. In other words, their degree of interaction is low, which traduces the fact that the economic environment and in particular the monetary environment is instable. According to the historical events, during this period, this result appears to be very significant. Indeed, the end of the 1960 s and the beginning of the 1970s was known as a period of great instability. Many shocks affected the economy. Indeed, at the beginning of the 1970s, the Bretton Woods system was brought down. In addition, in 1973, a hard oil shock affected the entire world economy. So, following these events, we can explain the low level of interaction between these monetary variables.

In 1975, many monetary measures were undertaken to cope with these shocks. Indeed, the policy-makers adopted a monetarist monetary policy ${ }^{18}$ based on the control of prices and wages. Targeting M1 concretized this approach. It succeeded in the medium run in reducing the volatility of some economic indicators, ${ }^{19}$ but not in the long run. We interpret the structural break identified in 1975 in the long-run cohesion, between the inflation rate, interest rate and GDP growth, as being the monetary policy change that occurred at that date. We consider that the relative increase in the cohesion since 1975 from 0.12 to 0.22 was the result of the adoption of a new monetary policy in 1975 (targeting M1).

The case of the United Kingdom is similar to the one of Canada. Indeed, we find that the coherence has a low level (0.15) during the 1960s. We interpret this as the performance deterioration of the British economy at the end of the 1950s. This is a result of a Keynesian vision followed in this period in the United Kingdom. According to Olivier and Pemberton (2006), this Keynesian vision generated a destabilization of the economy. In the case of an economic destabilization, it's fully logical to find a divergence between economic series. So, the main object during the 1960s was to modernize the economy. New economic objects and new instruments were adopted, targeting the long-run growth rate. In addition, they adopted a change in fiscal policy and some other reforms were undertaken during this period (like the devaluation of sterling in 1967...). All these actions contributed to a new economic framework, but this didn't succeed very well because it was subjected to some economic shocks. So, we interpret the first break point in the cohesion series, for the case of the United Kingdom, identified in the first quarter of 1971, as the instauration of this new economic framework. This generates a relative increase in the relationship between the three time series, from 0.15 to 0.24 . However, we suggest that the economic shocks have limited the high degree of convergence between economic series. Indeed, this new framework was subjected to some economic shocks, such as the collapse of the Bretton Woods regime, which destabilized the world monetary environment, and the oil shock.

The more interesting results concern the break point identified just before the inflation targeting adoption. For the cases of Canada and the United Kingdom, according to Tabs. 1 and 3, we identify a structural break point respectively in the first quarter of 1989 and in the first quarter of 1990. Since this date, the degree of interaction between economic series has increased respectively from 0.22 to 0.37 and from 0.24 to 0.4 . This result traduces a high degree of convergence between the inflation rate, interest rate and GDP growth in the long term. In other words, in the long term, there exists an equilibrium in which these variables converge. This high degree of interaction and convergence traduces that the monetary environment is certain and that the variables are predictable in the long term. This economic certainty and the high degree of interaction traduce the economic performance during this period. According to the analysis

\footnotetext{
${ }^{18}$ The rule of $K \%$ proposed by Friedman (1959).

${ }^{19}$ See Bordo and Redish (2005).
} 
of the economic events, we interpret these results as the effect of the inflation targeting policy. Before the 1990s, Canada and the United Kingdom adopted many monetary policies ${ }^{20}$ with an eye to achieving a stable macroeconomic environment that encourages investment and consumption and generates a suitable growth rate. However, these monetary policies didn't succeed in reaching this objective. In the case of Canada, from 1988, many seminars and reforms have been undertaken to study the possibility of adopting inflation targeting as a monetary policy. ${ }^{21}$ Many actions have been undertaken with the aim of raising the credibility and the transparency of the policy-makers' actions, such as the measure allowing more interdependence to the central Bank of Canada. Ftiti and Essaadi (1998) showed a structural break at the date of 1988 in the Canadian inflation series, which traduces long-term stability. They interpret this break as the inflation targeting implementation and the preparation of a favourable climate to implement this policy. Their conclusion is in line with that of Artus (2004) and the work of Ravenna (2004). As with these studies, our empirical work proves a change in the degree of long-run interaction between the three economic series, during the period of inflation targeting preparation. So, we conclude that inflation targeting generates a stabilization monetary environment, which encourages investment, consumption and production. These effects contribute to the economic performance caused by the inflation targeting policy.

The results in the case of the United Kingdom are similar to those of Canada. Indeed, we find a break point in the first quarter of 1990 (2 years before the inflation targeting adoption). The degree of the long-run interaction between the economic series we are studying increases from 0.24 to 0.4 . This rise generates a convergence of economic series and more certitude on the monetary environment, which positively affect the long-run growth. So, since this date, we can conclude that the monetary environment has generated a macroeconomic performance. In line with the analysis of Canadian cases, we explain this change in the nature of the relationship between economic series by the effect of the inflation targeting policy. Indeed, many reforms have been undertaken since 1988 to implement an exchange rate policy, such as financial deregulation, banking reforms and credit market deregulation... These reforms have as their objective the exchange rate policy implementation. According to Bean (2003), the frameworks of the inflation targeting policy and the exchange rate policy implemented in 1988 are the same. That's why we find no other reforms just before the inflation targeting adoption. Since the adoption of this policy, the relationship between economic variables has remained at a high level. So, we conclude that the inflation targeting policy succeeds in maintaining a stable monetary environment that favours suitable long-run growth. Thus, the inflation targeting policies contribute to a macroeconomic performance.

In our study, we present the result for the medium term ( 5 years) just for comparison with the long term. According to Tabs 2 and 4, the medium-term interactions between the economic variables remain relatively lower than those in the long run. So, we conclude that inflation targeting generates a more stable economic environment in the long run than in the medium run.

\footnotetext{
${ }^{20}$ Some monetary policies such targeting monetary indicators, targeting growth rate and exchange rate (fixed and flexible)...

${ }^{21}$ See McCallum (1996), Dodge (2002) and Ravenna (2002).
} 


\section{Conclusion}

In this paper, we try to check the macroeconomic performance of the inflation targeting policy. We differ from the previous studies along two lines. First, the methodology we follow is different. In our analysis, we consider the inflation targeting policy as a relevant policy when it generates a long-term stabilization in the monetary environment. This idea comes from the literature on the interaction between stabilization (or cycle volatility) and long-run growth. The latter indicator is considered in the literature as a proxy of the macroeconomic performance. The inflation targeting policy was traduced by a reaction function that has a Taylor-style rule. As shown by Ftiti (2008), this rule includes, in most of the cases of industrialized countries, three variables: inflation rate, interest rate and GDP growth. So, to measure the degree of stabilization in this monetary environment, we focus on the degree of interaction between these three economic series. The degree of interaction is measured by the notion of cohesion. This measure corresponds to the co-movement notion in the case of a bivariate process. To determine this cohesion, we refer to evolutionary co-spectral theory as defined by Priestley and Tong (1973), which allows us to have a time-varying measure of cohesion. After that, we apply the Bai and Perron test (19982003b) to detect the occurrence of a significant change in these series. The result shows that the degree of interaction increases in the case of Canada and the United Kingdom two years before the inflation targeting adoption. According to an economic events analysis during this period and according to the results of Artus (2004) and Ftiti and Essaadi (2008), we conclude that the transition period of inflation targeting generates a significant change in the degree of convergence between economic series. So, we conclude that, during this period and in the whole periods of ITP, the monetary environment is stable. So, this monetary policy generates a good economic performance in these countries.

In a further investigation, we suggest checking the relevance of the inflation targeting policy in the case of emerging countries. Indeed, this sort of country has additional institutional problems to industrialized countries. So, we are wondering if these results are extendable to emerging countries or not. 


\section{Bibliographie}

Ahamada I. Boutahar M. (2002). Tests for covariance stationarity and white noise, with application to Euro/US Dollar exchange rate. Economics letters, 77, pp. 177-186.

Ahamada B. Ben Aïssa M.S. (2004). Testing Multiple Structural Changes in US Output Gap Dynamics: Non-Parametric Approach. Finance India, Vol. XVIII, pp. 577-586.

Aizennman, J. M. Nancy (1993). Policy uncertainty, persistence and growth. Review of international economics, vol. 1, No. 2, p. 145-163.

Artus P. (2004). Le targeting (ciblage) d'inflation par les Banques centrales des PECOs est-il une bonne idée? Flash CDC-IXIS, n 2004-06

Ball, L. Sheridan, N. (2003). Does inflation targeting matter? IMF working paper, No. 03. 129. Bai, J. Perron, P. (1998). Estimating and Testing Linear Models with Multiple Structural Changes. Econometrica, 66, pp.47-78.

Bai, J. P. Perron, (2003a). Computation and Analysis of Multiple Structural Change Models. Journal of Applied Econometrics, 18, pp.1-22.

Bai, J. P. Perron, (2003b). Critical Values for Multiple Structural Change Tests. Econometrics Journal, 1, pp.1-7.

Bean, C. (2003). Inflation targeting The UK experience. Bank of England Quarterly Bulletin, pp. 479-494.

Beaudry, P., Caglayan, M et Schiantarelli, F. (2001). Monetary instability, the predictability of prices, and the allocation of investissement: An Empirical investigation using U.K Panel data. The American economic review, vol. 91, No. 3, p. 648-662.

Bernanke B.S., Laubach T., Mishkin F.S. Posen, A.S. (1999). Inflation Targeting: Lessons from the international experience. Princeton University Press: New Jersey.

Buckle, R. A., Kim, K McLellan, N. (2003).The impact of monetary policy on New Zealand business cycles and inflation variability. New Zealand treasury working paper, No. 03/9.

Black, Fisher (1987). Business cycles and equilibrium. Cambridge, M A: Blackwell.

Blackburn, K. Pelloni, A. (2005). Growth, cycles and stabilization policy. Oxford Economic Papers, forthcoming.

Canton, E. (1996). Business cycle in a two-sector model of endogenous growth. mimeo, Centre for Economic Research, University of Tilburg.

Caporale, Tony McKiernan, B. (1996). The Relationship between Output Variability and Growth: Evidence from Post War UK Data. Scottish Journal of Political Economy, Scottish Economic Society, vol. No 43, pages 229-36.

Caballero R. and HammourM. (1994). The Cleansing Effect of Recession. American Economic Review, n 84(5), pp. 1350-1368.

Croux, C., Forni, M Reichlin, L. (2001). A measure of comovement for economic variables: Theory and empirics. The review of economics and statistics, vol. 83, No. 2, p. 232-241.

Elbadawi, I Schmidt-Hebbel, K (1998). Macroeconomic policies, Instability, and growth in the world. Documentos de trabajo del banco central, $\mathrm{N}^{\circ} 43$.

Essaadi, E. Boutahar, M. (2008). A Measure of Variability in Co-movement for EconomicVariables: a Time-Varying Coherence Function Approach. Gate working paper, No. 08/27.

Fischer, S, (1993). The Role of Macroeconomic Factors in Growth. Journal of Monetary Economics. Vol. 32, No 5, p. 455-511.

Ftiti, Z. (2008). Taylor Rule And Inflation Targeting: Evidence From New Zealand. Interna- 
tional Business and economic research, vol7, issue1. pp. 131-150.

Ftiti, Z Essaadi, E. (2008). The inflation Targeting effect on the inflation series: ANew Analysis Approach of evolutionary spectral analysis. GATE working paper, No. 08/32.

Grier, K. B G. Tullock. (1989). An empirical analysis of cross-national economic growth, 195180. Journal of monetary Economics, vol. 24. No. 2, p. 259-276.

Ho, W-M. (1996). The imperfect information, money and economic growth. Journal of money, credit and banking, vol 28, No. 4, p. 578-603.

Honda Y. (2000). Some tests on the effects of inflation targeting in New Zealand, Canada, and the UK. Economics Letters, 66 (2000).pp.1-6

Ismihan, B., Metin-Ozcan, K. Transel, A. (2003). Macroeconomic instability, capital accumulation and Growth: the case of Turkey 1963-1999. METU working paper, $\mathrm{N}^{\circ} 2$. http://www.erc.metu.edu.tr/menu/series Kneller, R. et Young, G. (2001). Business cycle volatility, uncertainty and long-run growth. Manchester School, 69, 534-52.

Kormendi, Roger C., P. Meguire. (1985). Macroeconomic determinants of growth: cross-country evidence. Journal of Monetary economics, vol. 16. No. 2, p. 141-164.

Kydland, F. y E. Prescott (1982). Time to build and aggregate fluctuations. Econometrica, vol. 50, No. 6, p. 1345-70.

Lensink R., Bo H. and Sterken E. (1999) "Does Uncertainty Affect Economic Growth? An Empirical Analysis", Weltwirtschaftliches Archiv, 135, 379-396

Liu J., Wu S. Zideck J.V. (1997). On segmented multivariate regressions. Statistical Sinica, 7, 497-525

Martin, P. Rogers, C.A (2000). Long term growth and short term economic instability. European economic review, 44, p. 359-81.

Mishkin F.S. Posen A.S. (1997). Inflation Targeting: Lessons from Four Countries. Economic Policy Review, Federal Reserve Bank of New York 3, 9-110.

Mollick, V., Torres, A., Cabral, R Carneiro, F. G. (2008). Does Inflation Targeting Matter for Output Growth? Evidence from Industrial and Emerging Economies. World Bank policy Research Working Paper Series, No. 4791.

Nelson C. Plosser C. (1982). Trends and Random Walks in Macroeconomic Time series: Some evidence and implications. Journal of monetary economics, No. 10.

Olivier. M. J Pemberton, H. (2006). UK economy policy in the 1960 and 1970 and the challenge to learning. http://www.helsinki.fi/iehc2006/papers1/Oliver19.pdf

Perron, P.(1997). Further evidence on breaking trend functions in macroeconomic variables. Journal of econometrics, 80, pp. 335-385.

Priestley, M. B. (1965). Evolutionary spectra for non stationary process. Journal of royal statistic society. ser. B, 27, p. 204-237.

Priestley, M. B. (1966). Design relations for non stationary processes. Journal of royal statistic society. ser. B, 28, p. 228-240.

Priestley M. B. (1969). A Test for Non-Stationary of Time Series. Journal of Royal Statistical Society, 31, pp. 140-149.

Priestley, M. B. Tong, H. (1973). On the analysis of bivariate non-stationary processes. Journal of royal statistic society. ser. B, 35, p. 135-166.

Priestley, M. B. (1981). Spectral Analysis and Time Series. Academic Press, New York. Priestley M. B. (1988). Non-Linear and Non-Stationary Time Series Analysis. Academic Press, London. 
Priestley M. B. (1996). Wavelets and time-dependent spectral analysis. Journal of Time Series Analysis, 17, 1, pp. 85-103.

Ramey G. Ramey V.A. 1995. Cross-Country Evidence on the link between volatility and growth. American economic review. No. 5.

Sanchez-Robles, B. (1998). Macroeconomic stability and economic growth; the case of Spain. Applied economic letters, vol 5, p. 587-591.

Stiroh, K. J. (2006). Volatility accounting: A production perspective on increased economic instability. FRBNW staff reports, No. 25.

Turnovsky, S. J. (2003). Macroeconomic policies, Growth, and welfare in stochastic economy. International economic review, vol. 34, No. 4, p. 953-981.

Winkler, B. (2003). Stability and Growth: The role of the monetary policy and other policy actors in EMU. 


\section{Appendix 1}

Table 1: Cohesion between the interest rate, inflation rate and GDP growth for the frequency $\frac{\pi}{20}$ : the case of Canada.

\begin{tabular}{llll}
\hline \hline Estimators & $T_{1}$ & $T_{2}$ & \\
Break Date & $1975 \mathrm{Q}: 1$ & $1989 \mathrm{Q}: 1$ & \\
Coefficient & 0.12 & 0.22 & 0.37 \\
Standard Error & 0.005 & 0.004 & 0.004 \\
\hline \hline
\end{tabular}

Table 2: Cohesion between the interest rate, inflation rate and GDP growth for the frequency $\frac{4 \pi}{20}$ : the case of Canada.

\begin{tabular}{llll}
\hline \hline Estimators & $T_{1}$ & $T_{2}$ & \\
Break Date & $1990 \mathrm{Q}: 4$ & $1998 \mathrm{Q}: 2$ & \\
Coefficient & 0.08 & 0.20 & 0.31 \\
Standard Error & 0.004 & 0.01 & 0.01 \\
\hline \hline
\end{tabular}

Table 3: Cohesion between the interest rate, inflation rate and GDP growth for the frequency $\frac{\pi}{20}$ : the case of United Kingdom.

\begin{tabular}{llll}
\hline \hline Estimators & $T_{1}$ & $T_{2}$ & \\
Break Date & $1971 \mathrm{Q}: 1$ & $1990 \mathrm{Q}: 1$ & \\
Coefficient & 0.125 & 0.24 & 0.39 \\
Standard Error & 0.006 & 0.006 & 0.006 \\
\hline \hline
\end{tabular}

Table 4: Cohesion between the interest rate, inflation rate and GDP growth for the frequency $\frac{4 \pi}{20}$ : the case of the United Kingdom.

\begin{tabular}{llll}
\hline \hline Estimators & $T_{1}$ & $T_{2}$ & \\
Break Date & $1984 \mathrm{Q}: 1$ & $1994 \mathrm{Q}: 2$ & \\
Coefficient & 0.11 & 0.06 & 0.16 \\
Standard Error & 0.004 & 0.01 & 0.01 \\
\hline \hline
\end{tabular}




\section{Appendix $2^{22}$}

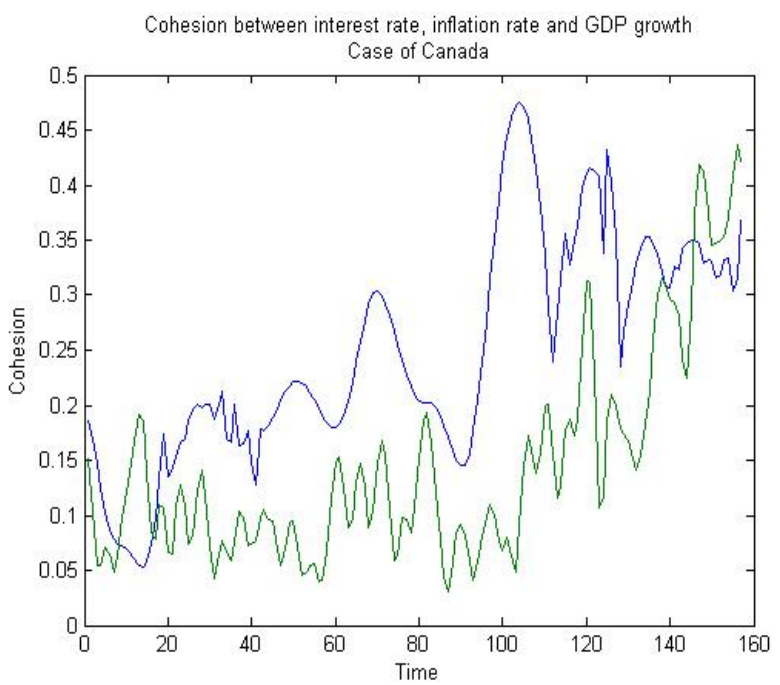

Figure 1: Cohesion in two frequencies (Long-term and medium-term) between the interest rate, inflation rate and GDP growth: the case of Canada.

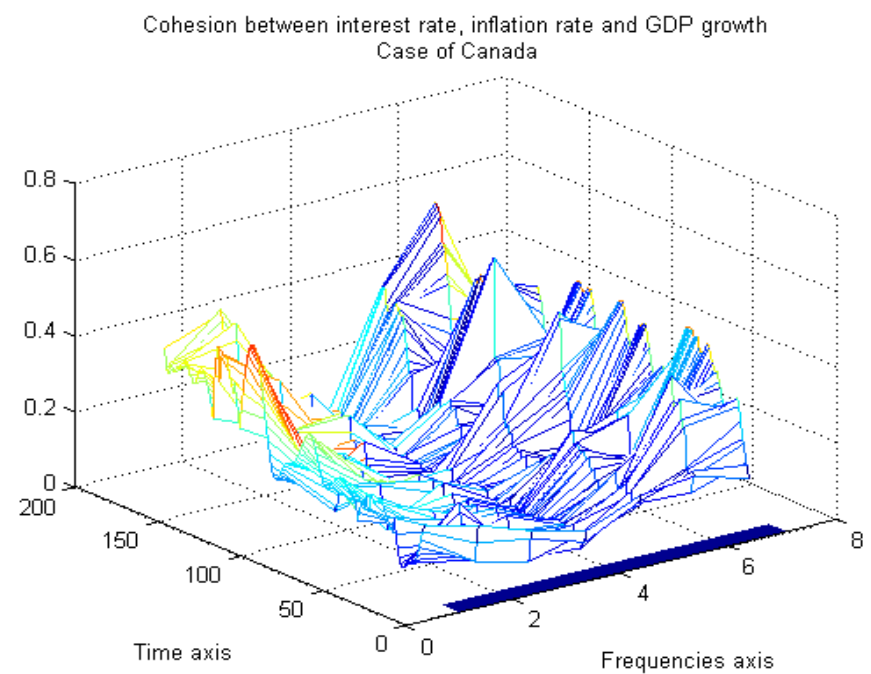

Figure 2: Cohesion in all frequencies between the interest rate, inflation rate and GDP growth: the case of Canada.

\footnotetext{
${ }^{22}$ the blue line correspond to long-run cohesion and the green correspond to the medium term
} 


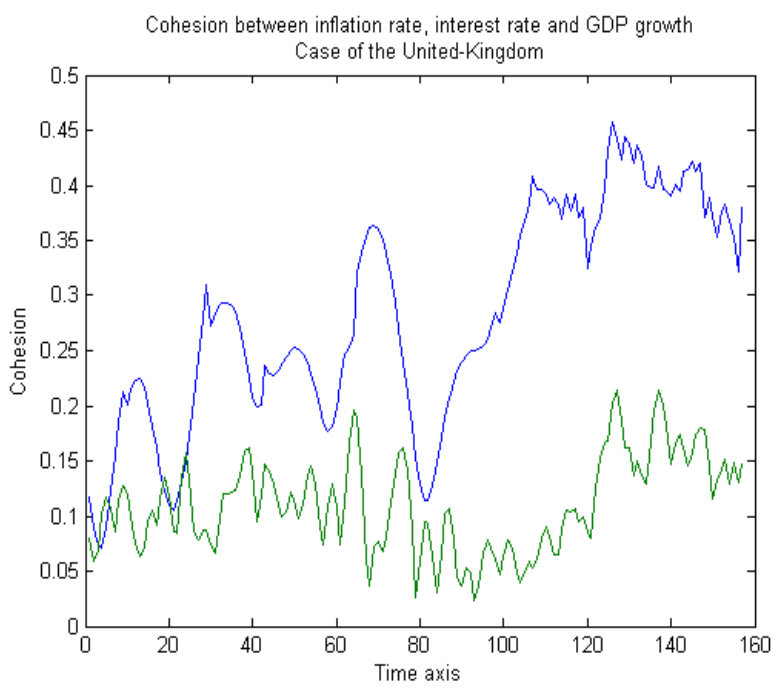

Figure 3: Cohesion in two frequencies (Long-term and medium-term) between the interest rate, inflation rate and GDP growth: the case of United Kingdom.

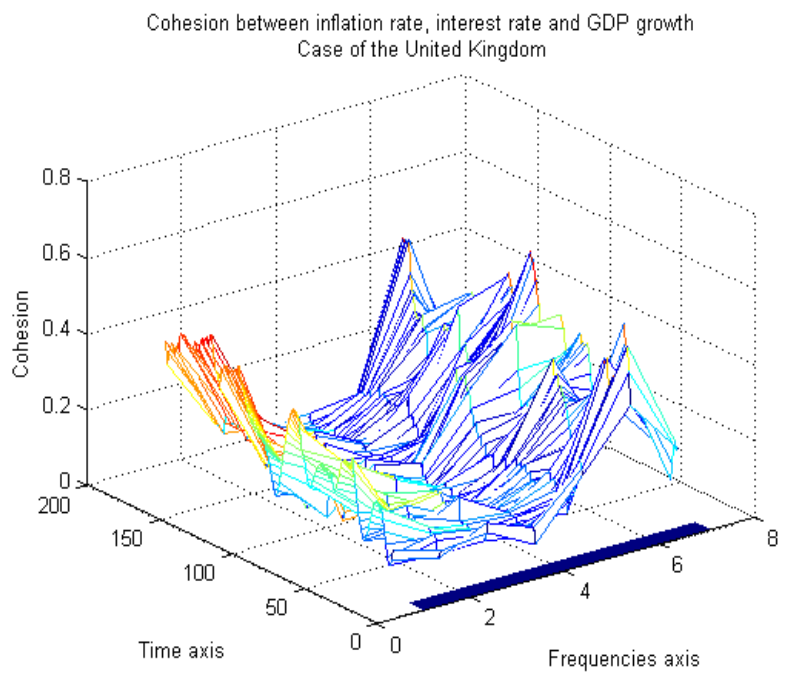

Figure 4: Cohesion in all frequencies between the interest rate, inflation rate and GDP growth: the case of United Kingdom. 